\title{
EVIDÊNCIAS SEDIMENTARES DE ANTIGO AMBIENTE MARINHO, MONTANHAS ELLWORTH, ANTÁRTICA OCIDENTAL
}

\author{
Rosemary Vieira $^{(a)}$, Vanessa do Couto Silva Costa ${ }^{(b)}$, Matheus Gonçalves ${ }^{(c)}$, João Vitor Santos \\ Ramos $^{(\mathrm{d})}$, João Pedro Farias Santos ${ }^{(\mathrm{e})}$, Marcos Aurélio Perroni ${ }^{(\mathrm{f})}$
}

(a) Laboratório de Processos Sedimentares e Ambientais (LAPSA), Departamento de Geografia/Instituto de Geociências, Universidade Federal Fluminense, e-mail: rosemaryvieira@id.uff.br

(b) Laboratório de Processos Sedimentares e Ambientais (LAPSA), Departamento de Geografia/Instituto de Geociências, Universidade Federal Fluminense, e-mail: vanessacosta@id.uff.br

(c) Laboratório de Processos Sedimentares e Ambientais (LAPSA), Departamento de Geografia/Instituto de Geociências, Universidade Federal Fluminense, e-mail: matheusgoncalves12@ hotmail.com

(d) Laboratório de Processos Sedimentares e Ambientais (LAPSA), Departamento de Geografia/Instituto de Geociências, Universidade Federal Fluminense, e-mail: jv.ramos@ @ive.com

(e) Laboratório de Processos Sedimentares e Ambientais (LAPSA), Departamento de Geografia/Instituto de Geociências, Universidade Federal Fluminense, e-mail: ipfariasantos93@gmail.com

${ }^{(f)}$ Laboratório de Processos Sedimentares e Ambientais (LAPSA), Departamento de Geografia/Instituto de Geociências, Universidade Federal Fluminense, e-mail: perroni_elio@hotmail.com

\section{Eixo: GEOCRONOLOGIA E ESTUDOS PALEOAMBIENTAIS}

\begin{abstract}
Resumo
O vale Elephant Head $\left(79^{\circ} .298^{\prime} \mathrm{S} / 83^{\circ} 20.426^{\prime} \mathrm{W}\right)$ é um vale deglaciarizado no qual amostras sedimentares foram coletadas em depósitos morâinicos. Análise do tamanho e morfometria das partículas, composição química e mineralógica foram efetuadas. O predomínio de carbonato de cálcio foi observado na maioria das amostras. Marcas onduladas (ripple marks) sugerem a existência de ambiente marinho raso. Um processo de sedimentação marinha preencheu possivelmente esse mar interior, associado com soerguimento tectônico local, posterior cobertura e ação glacial. Sedimentação calcária e clástica domina o vale, refletindo a natureza e composição das principais fontes de sedimentos. Os depósitos e sedimentos no vale Elephant Head corroboram com outros estudos que caracterizam a área como uma zona de rift em um ambiente sedimentar marinho raso. A principal lacuna é o timing desses eventos.
\end{abstract}

Palavras chave: Antártica; Paleoclimatologia; Geomorfologia Glacial; Sedimentologia.

\section{Introdução}

As Montanhas Ellsworth são caracterizadas por numerosos vales formados pela erosão glacial e têm uma elevada concentração de depósitos superficiais e feições erosivas, as quais contêm registros geológicos, climáticos e da história ambiental. As Montanhas Ellsworth formam uma cadeia localizada cerca de $79^{\circ} \mathrm{S}$ $84^{\circ} \mathrm{W}$ e de aproximadamente $415 \mathrm{~km}$ de extensão no sentido NNE-SSW (Tabela 1) e estão divididas em dois setores geograficamente distintos: a Cadeia Sentinel, ao norte, e a Cadeia Heritage, ao sul (DENTON et al., 1992). As montanhas estão situadas ao longo da periferia norte do bloco crustal Ellsworth- 


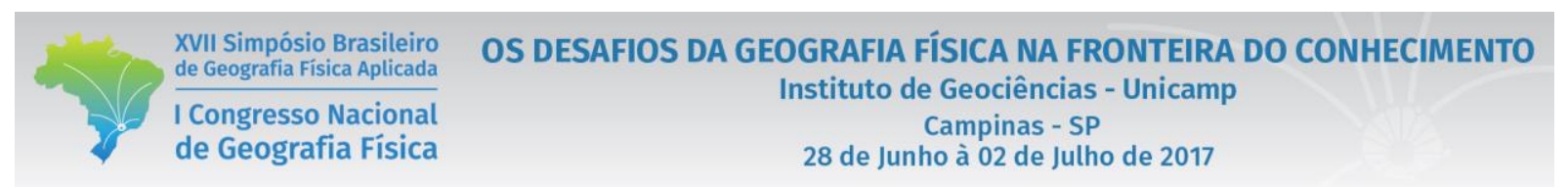

Whitmore, que representa parte do terreno deslocado situado ao longo da margem Pacífica do Gondwana, antes da ruptura do supercontinente (CURTIS et al., 1999; STOREY, 2005). O estilo tectônico dessa margem variou tanto temporal como espacialmente durante esse período, com regiões de deformação compressional associada a eventos de subducção, coexistindo com plataformas de sedimentação carbonática e tectônica extensional (STUMP, 1995).

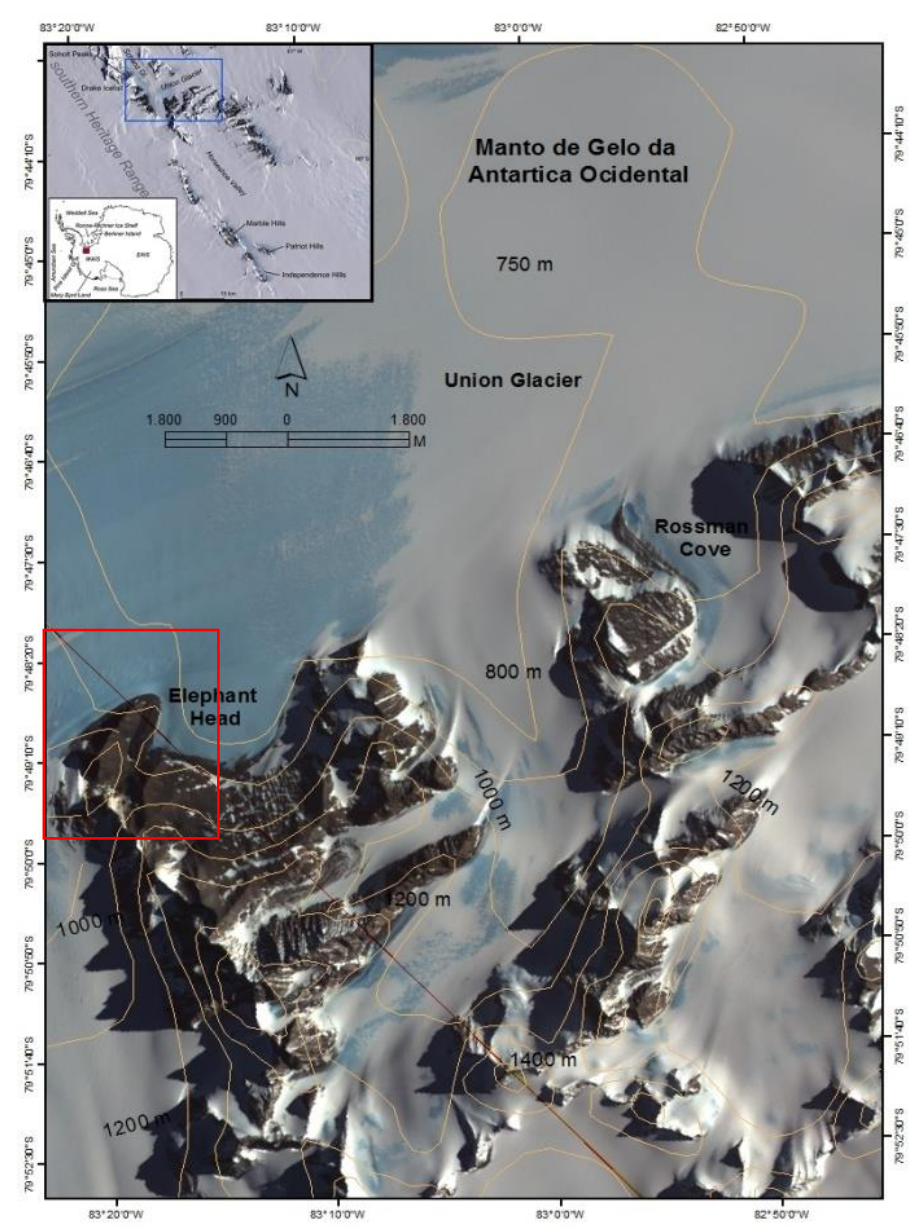

Figura 1 - Localização da área de estudo (quadrado).

Sucessões do Paleozóico Inferior nas Montanhas Ellsworth compreendem o Grupo Heritage (Cambriano Inferior ao Superior - 7,5 km de espessura), o qual é coberto pelo Grupo Crashsite (Cambriano Superior ao Devoniano - $3 \mathrm{~km}$ de espessura). O Grupo Heritage é composto predominantemente por rochas sedimentares clásticas e vulcânicas que compreendem mais da metade da espessura estratigráfica das Montanhas Ellsworth. As rochas vulcânicas foram formadas no rift continental do Cambriano Médio, com erupções próximas ao eixo do rift. A deposição do Grupo Heritage ocorreu no Cambriano Médio e Tardio em uma bacia subsidente bordeada por sucessões de rochas carbonáticas e quartzíticas (WEBERS et al., 1992a). O Grupo Heritage é recoberto por $3 \mathrm{~km}$ de quartzitos do Grupo Crashsite, diamictitos Permo- 
Carbonífero do Conglomerado Whiteout e da Formação Permiana Polar Star (STOREY, 2005). Sequência de depósitos fluviais e sedimentos marinhos deltaicos são encontrado na área de Edson Hills (WEBER et al., 1992a).

Na Cadeia Heritage são encontrados clastos carbonáticos nas Formações Union Glacier e Conglomerate Ridge, do Cambriano Médio, e na Formação Whiteout, do Permo-Carbonífero. Arenitos interlaminados com xistos são encontrados nas Formações Drake Icefall e Minaret, do Cambriano Médio. Rochas carbonáticas ocorrem nos Picos Soholt (Formação Conglomerates Ridge). Tais conglomerados refletem áreas fontes próximas de relevo moderado e representam deposição fluvial e/ou marinha rasa. Outras unidades carbonáticas são encontradas em Webers Peak, Springer Peak, Bingham Peak, Mount Dolence e em Marble Hills. A Formação Minaret é a unidade carbonática mais proeminente nas Montanhas Ellsworth (BUGGISH e WEBERS, 1992).

No verão de 2011-12, durante as atividades do Instituto Nacional de Ciência e Tecnologia da Criosfera no manto de gelo da Antártica Ocidental - Expedição Criosfera, a área da geleira Union foi estudada com incursões em vários setores isolados, entre eles o vale Elephant Head. Portanto, o objetivo desse trabalho é mostrar evidências sedimentológicas inéditas de um paleoambiente marinho no vale Elephant Head, setor sul das Montanhas Ellsworth.

\section{2. Área de Estudo}

Elephant Head é um vale tributário deglaciarizado da geleira Union, localizado no setor sul da cadeia Heritage. A geleira Union flui em direção à Plataforma de gelo Ronne e está estável durante as últimas décadas (STEIG et al., 2009). A geleira União é alimentada por diversas geleiras tributárias, sendo as geleiras Union e Schanz as principais. Estas fluem do interior do platô polar antes de se unirem no setor mais estreito da geleira, o qual é caracterizado por uma extensa área de gelo-azul e uma moraina central (RIVERA et al., 2010). Com exceção de algumas geleiras de vale, cujas áreas marginais são terrestres, a maioria drena para o fluxo principal da geleira Union. A principal feição na área é o Monte Dolence, no flanco leste do vale Elephant Head, que incorpora a Formação Minaret em sua porção sul. Sua espessura aumenta em direção sul, de 8m em Webers Peaks a centenas de metros na área de Marble Hills (CURTIS e LOMAS, 1999).

\section{Procedimentos Metodológicos}


Análises de formas glacialmente modificadas na geleira Union foram feitas a partir dos trabalhos de campo e materiais coletados durante a expedição Criosfera, realizada no verão de 2011-12.

As amostras foram coletadas com uma pá, em pequenas cavidades, a fim de evitar o material meteorizado da superfície, na quantidade de 100 gramas e postas em 6 sacos plásticos, contendo o código de cada amostra. Em cada local de coleta foram realizados pontos de controle de GPS (altitude e coordenadas, com margem de erro de 3 metros - Tabela 1) e fotografias com escala e observação do ambiente de entorno (orientação, tamanho do depósito, matriz). Também foram coletados 50 clastos por amostra, com eixo maior > $15 \mathrm{~mm}$ para análise morfométrica.

Tabela 1. Localização dos pontos de amostragem de sedimentos.

\begin{tabular}{|c|c|c|c|}
\hline Amostra & Latitude & Longitude & Altitude \\
\hline EH-1 & $79^{\circ} 49.298^{\prime}$ & $83^{\circ} 20.426^{\prime}$ & 810 \\
\hline EH-2 & $79^{\circ} 49.238^{\prime}$ & $83^{\circ} 18.928^{\prime}$ & 739 \\
\hline EH-3 & $79^{\circ} 49.131^{\prime}$ & $83^{\circ} 20.425^{\prime}$ & 691 \\
\hline EH-4 & $79^{\circ} 49.289^{\prime}$ & $83^{\circ} 20.020^{\prime}$ & 741 \\
\hline EH-5 & $79^{\circ} 49.229^{\prime}$ & $83^{\circ} 20.077^{\prime}$ & 700 \\
\hline EH-6 & $79^{\circ} 49.032^{\prime}$ & $83^{\circ} 20.032^{\prime}$ & \\
\hline
\end{tabular}

A distribuição do tamanho das partículas de cada amostra foi determinada em laboratório por peneiramento a seco com intervalos em phi, de $-3 \varnothing$ a $4 \varnothing(8 \mathrm{~mm}$ a $0,063 \mathrm{~mm})$. A análise da forma dos clastos (arredondamento) e a descrição das características superficiais (estrias e polimentos) foram feitas em lupa binocular a fim de analisar as fácies dos depósitos. 50 clastos de cada amostra foram mensurados manualmente usando caliper digital para determinar visualmente o tamanho relativo dos três eixos ortogonais: $a$ (maior), $b$ (intermediário) e $c$ (menor).

Os valores de arredondamento dos clastos (de 2 a $8 \mathrm{~mm}$ ) foram assignados usando o critério descritivo de Benn e Ballantine (1994), modificado de Powers (1953). Em adição, a análise de co-variância do índice $\mathrm{C}_{40}$ (\% de clastos com eixo $\left.c / a=0,4\right)$ e índice RA (\% de clastos muito angulosos e angulosos) foi conduzida de acordo com o método proposto por Benn e Balllantyne (1994) e Evans e Benn (2004). Esse método distingue os sedimentos que foram transportados ativamente daqueles que o foram de forma passiva pela geleira (BENN e BALLANTYNE, 1994; BENNETT et al., 1997; LUKAS et al., 2013). 
Diagramas ternários e histogramas, produzidos através do software Triplot, apresentam a forma e o arredondamento dos clastos coletados.

Para as análises litológicas foram utilizados 200 clastos acima de $>2 \mathrm{~cm}$ de cada amostra, em lupa binocular e subdivididas baseadas em suas propriedades litológicas. As concentrações dos elementos principais e traços foram determinadas por espectroscopia de raio $\mathrm{X}$ por energia dispersiva (EDXRF), usando SHIMADZU EDX-720, em frações de 0,063 mm. As amostras foram preparadas no Laboratório de Sedimentologia do Instituto de Geociências e analisadas no Laboratório de Reatores, Cinética e Catálise, do Departamento de Engenharia Química, Universidade Federal Fluminense.

A composição mineralógica foi determinada com a mesma alíquota de sedimento por Difratometria de raio X usando o difratômetro Brucker D8 (XRD) no Laboratório de Difração de Raio X, Instituto de Física, Universidade Federal Fluminense. Cada amostra foi escaneada de $2 \theta$ a $70^{\circ} 2 \theta$ com radiação $\mathrm{CuK} \alpha$ (40 $\mathrm{kV}$ and $40 \mathrm{~mA}$, usando $0.02^{\circ} 2 \theta$ escaneamento passo a passo e $0.5 \mathrm{~s}$ por passo, com um detector LYNXEYE. Os minerais foram identificados a partir de seus picos a partir do software DIFRA EVA, versão 3, usando a base de dados minerais da USGS (United State Geological Survey).

\section{Resultados e Discussões}

Elephant Head é um vale deglaciarizado da geleira Union. Os depósitos morâinicos registram um antigo fluxo glacial pelo vale. Tem $1 \mathrm{~km}$ de extensão aproximadamente; o setor mais elevado e largo do vale está a 900 m, enquanto a zona de confluência com a geleira Union corresponde ao setor mais estreito, que é obstruído pelo cordão morâinico supraglacial desenvolvido na área de gelo-azul. O cordão morâinico tem alturas superiores a 7 metros e com o predomínio de cascalho e bloco. A moraina de núcleo de gelo tornase mais espessa em direção ao interior do vale, o que infere fluxo glacial em direção à essa margem, processos periglaciais com atividades de vertentes, ou a superfície do manto de gelo mais elevada Os sedimentos superficiais no vale e sobre parte das vertentes rochosas de ambos os lados do vale consistem de rochas de entorno intemperizadas, sedimentos marinhos e glaciais sujeitos aos processos periglaciais posteriores. Algumas formas de depósitos marinhos ocorrem em associação com os depósitos glaciais. Afloramentos e blocos bem expostos descrevem feições que são indicadores de processos deposicionais: marcas onduladas (ripple current) em arenitos e marcas onduladas assimétricas em quartzitos sobre arenitos com marcas onduladas de cristas planas. Blocos de arenitos cobrem de forma descontínua terrenos próximos às encostas, entre feições dômicas de carbonato, alguns dos quais com cristais de calcita incrustados, e domos de conglomerados . A Figura 2 mostra em detalhe a face de um bloco de arenito de 14 x $12 \mathrm{~cm}$ de dimensões, expondo bandas paralelas separada por surcos. As superfícies tanto das cristas 
como dos surcos são quase planas, com largura variando de 1,7 a $2 \mathrm{~cm}$. A distância entre as cristas é 1,5 $\mathrm{cm}$. As marcas onduladas apontam para um ambiente aquático e seus perfis simétricos sugerem um antigo mar raso, como o reportado por Curtis e Lomas (1999) para as fácies areníticas presentes na Formação Minarete entre Webers Peaks e Mount Dolence. Vialov (1962) descreveu marcas desse tipo em Beacon Height West, em Victoria Land. A identificação de fragmentos de rochas sedimentares contendo feições marinhas em e entre depósitos morâinicos indica a existência de outro estágio na cobertura sedimentar na área da geleira Union. Depósitos marinhos forma soerguidos e cobertos pelo gelo, e expostos nos depósitos morâinicos após a retração do gelo.

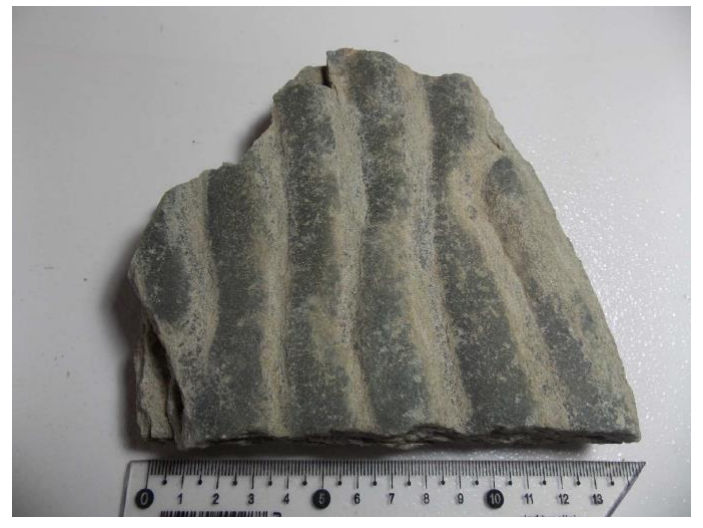

Figura 2. Vista de marcas onduladas sinuosas sobre bloco de arenito.

A Tabela 2 reúne dados estatísticos das amostras coletadas. Análise granulométrica mostram o predomínio de cascalho arenoso e areias. A proporção de partículas finas é muito baixa e o conteúdo da fração areia varia de 38 a 52\%. As paredes rochosas abrigam o vale e fornece material desde as encostas cobertas por quartzitos e arenitos, o que pode, portanto, explicar a grande quantidade de sedimentos de fração areia. Os ventos catabáticos constantes são responsáveis pelo deslocamento de material mais fino, o que foi reportado por Souza (2015) ao analisar solos deste mesmo vale.

A forma dos clastos é apresentada em diagramas ternários e o arredondamento em histogramas (Figura 3a). $\mathrm{O}$ índice $\mathrm{C}_{40}$ relativamente alto nos clastos $>2 \mathrm{~mm}$ provê a indicação de baixa modificação dos detritos, o que significa transporte passivo ou pequena distância de deslocamento pela geleira (BENN e BALLANTYNE, 1994). Clastos sub-angulares e sub-arredondados predominaram em todas as amostras, seguidos por clastos arredondados; clastos muito angulares e clastos bem-arredondados são raros ou ausentes (Figura 3b). Essas características podem constituir em evidências de duas situações prováveis: (1) do retrabalho de antigos sedimentos pela ação das ondas, o que indica deposição em um ambiente aquático não congelado; (2) ação intensa do vento sobre a região a partir do estabelecimento das 

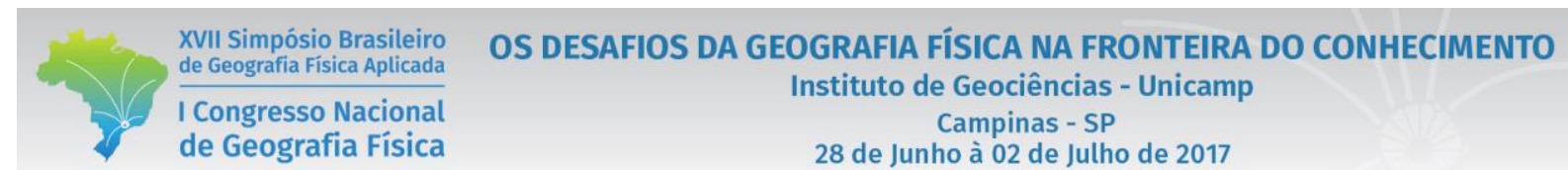

condições glaciais. Esses cenários distintos representam distintos períodos de deposição em ambientes aquático, glacial e terrestre, com retração da geleira ao longo do eixo do vale e mudanças morfológicas pós-deposicionais.

Tabela 2. Sumário da análise estatística das amostras morâinicas de Elephant Head. M= média; s= seleção.

\begin{tabular}{|c|c|ccc|cc|c|l|}
\hline \multirow{2}{*}{ Amostra } & \multirow{2}{*}{ Altitude } & \multicolumn{3}{|c|}{ Composição granulométrica } & \multicolumn{2}{c|}{$\begin{array}{c}\text { Parâmetros } \\
\text { granulométricos } \\
\text { (Folk e Ward, } \\
\text { 1957) }\end{array}$} & Seleção & \multicolumn{1}{c|}{$\begin{array}{c}\text { Descrição do } \\
\text { sedimento }\end{array}$} \\
\hline EH-1 & 810 & Cascalho & Areia & Silte & M & S & & Cascalho arenoso fino \\
\hline EH-2 & 739 & 47,4 & 52,5 & 0,1 & $-0,4$ & 1,8 & Moderado & $\begin{array}{l}\text { Cascalho arenoso muito } \\
\text { fino }\end{array}$ \\
\hline EH-3 & 691 & 58,2 & 41,8 & 0,0 & $-0,6$ & 1,4 & Pobre & Cascalho arenoso fino \\
\hline EH-4 & 818 & 70,1 & 29,5 & 0,4 & $-1,0$ & 1,1 & Moderado & Cascalho arenoso fino \\
\hline EH-5 & 741 & 51,0 & 48,9 & 0,1 & $-0,6$ & 1,9 & Pobre & Cascalho arenoso fino \\
\hline EH-6 & 700 & 61,2 & 38,6 & 0,2 & $-1,0$ & 1,7 & Pobre & Cascalho arenoso fino \\
\hline
\end{tabular}
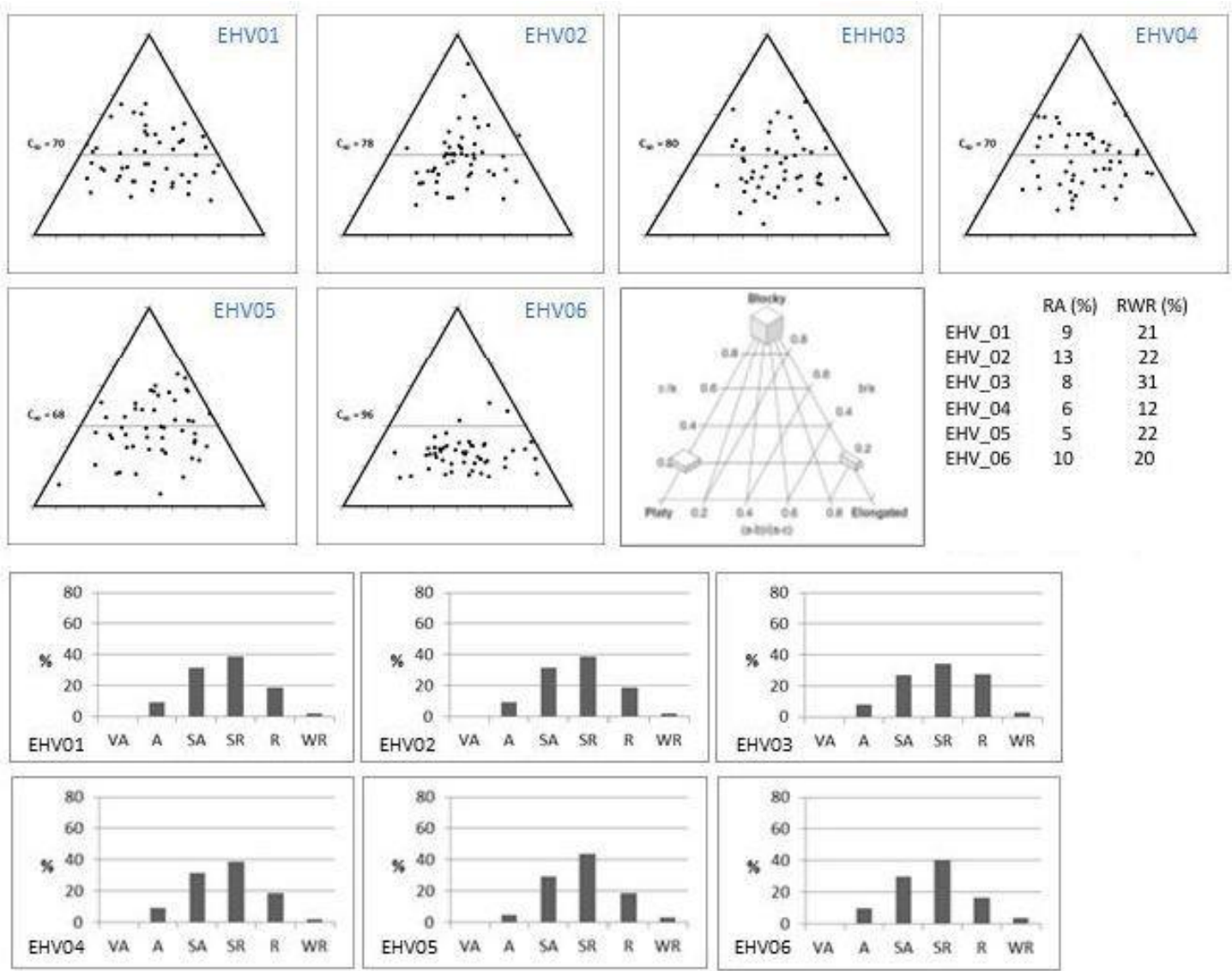

Figura 3. Dados morfológicos dos clastos: (a) diagrama ternário (forma do clasto); (b) histogramas (arredondamento). $\mathrm{O}$ índice $\mathrm{C}_{40}$ (percentual de clastos com razão $\mathrm{c}: \mathrm{a} \leq 0.4$ ), índice RA (percentual de clastos muito angulares), índice RWR 


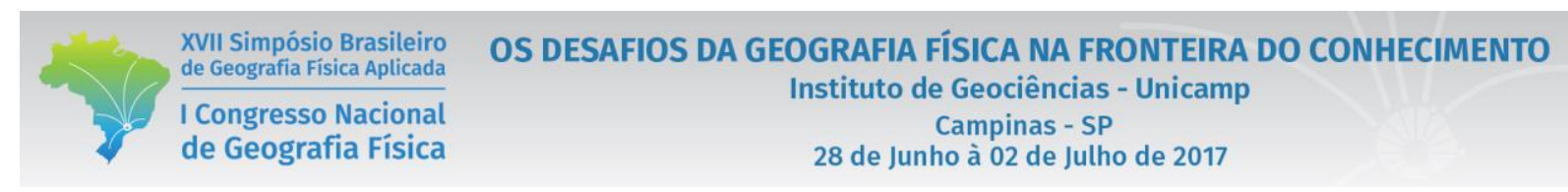

Análises das frações areia apontaram o domínio dos carbonatos (34\%), fragmentos de arenito (30\%) e quartzitos (28\%); fragmentos de conglomerados (4\%), grãos de quartzo (3\%) e outros fragmentos de rocha (1\%) apresentaram pouca participação. A fração silte compreende proporções similares. A maioria dos materiais depositados em Elephant Head Valley é derivada, portanto, de antigos processos marinhos. O menor percentual de carbonato (4\%) e o maior percentual de quartzito $(68 \%)$ foram observados na parte inferior do vale, onde ouros componentes mais modernos passaram a influenciar os processos de deposição, como as atividades glaciais e periglaciais.

A composição química dos sedimentos é apresentada na Tabela 3. Como o reportado por Souza (2015), intemperismo químico é baixo na área. Ca predomina, refletindo a contribuição do material carbonático parental (SPÖRLI, 1992), seguido por Si e Fe.

Tabela 3. Percentual de concentração dos elementos nos sedimentos.

\begin{tabular}{|c|c|c|c|c|c|c|c|c|c|c|c|c|c|}
\hline Amostra & $\mathbf{S i}$ & $\mathbf{A l}$ & $\mathbf{F e}$ & $\mathbf{C a}$ & $\mathbf{K}$ & $\mathbf{T i}$ & $\mathbf{M g}$ & $\mathbf{M n}$ & $\mathbf{S}$ & $\mathbf{R b}$ & $\mathbf{S r}$ & $\mathbf{Y}$ & $\mathbf{V}$ \\
\hline $\mathbf{E H - 1}$ & 8,19 & 3,43 & 5,89 & 78,15 & 2,53 & 0,73 & 0,57 & 0,32 & 0,07 & 0,07 & 0,16 & 0,10 & 0,03 \\
\hline $\mathbf{E H - 2}$ & 3,51 & 1,31 & 3,28 & 87,71 & 1,04 & 0 & 0,55 & 0,08 & 0 & 0 & 0 & 0,10 & 0 \\
\hline EH-3 & 6,36 & 2,23 & 4,46 & 84,34 & 1,70 & 0,54 & 0 & 0,09 & 0,08 & 0,08 & 0,08 & 0,10 & 0,02 \\
\hline EH-4 & 2,58 & 1,21 & 1,60 & 91,74 & 0,80 & 0 & 0 & 0,07 & 0 & 0 & 0 & 0,07 & 0 \\
\hline EH-5 & 4,10 & 1,51 & 2,23 & 90,58 & 1,10 & 0,27 & 0 & 0,05 & 0,06 & 0,06 & 0,06 & 0,09 & 0 \\
\hline EH-6 & 23,23 & 7,19 & 11,83 & 47,49 & 6,87 & 1,69 & 0,91 & 0,19 & 0,29 & 0,29 & 0,29 & 0,12 & 0,07 \\
\hline
\end{tabular}

A distribuição da composição mineralógica de material fino é apresentada na Figura 4. As espécies mineralógicas evidenciadas nas amostras morâinicas correspondem a três principais picos: carbonato de cálcio, calcita e quartzo. As frações areia mostram composição parental similar. Souza (2015) encontrou minerais de clorita, ilita, calcita e halita na fração argila das amostras superficiais de solo. A presença de calcita na fração argila é uma evidência da contribuição do material parental para a formação do solo no vale. Sub-populações de quartzo tanto nas frações areia como silte-argila podem ser indicativos da fonte de sedimentos, como o arenito (BASU, 1985) e quartzito. 

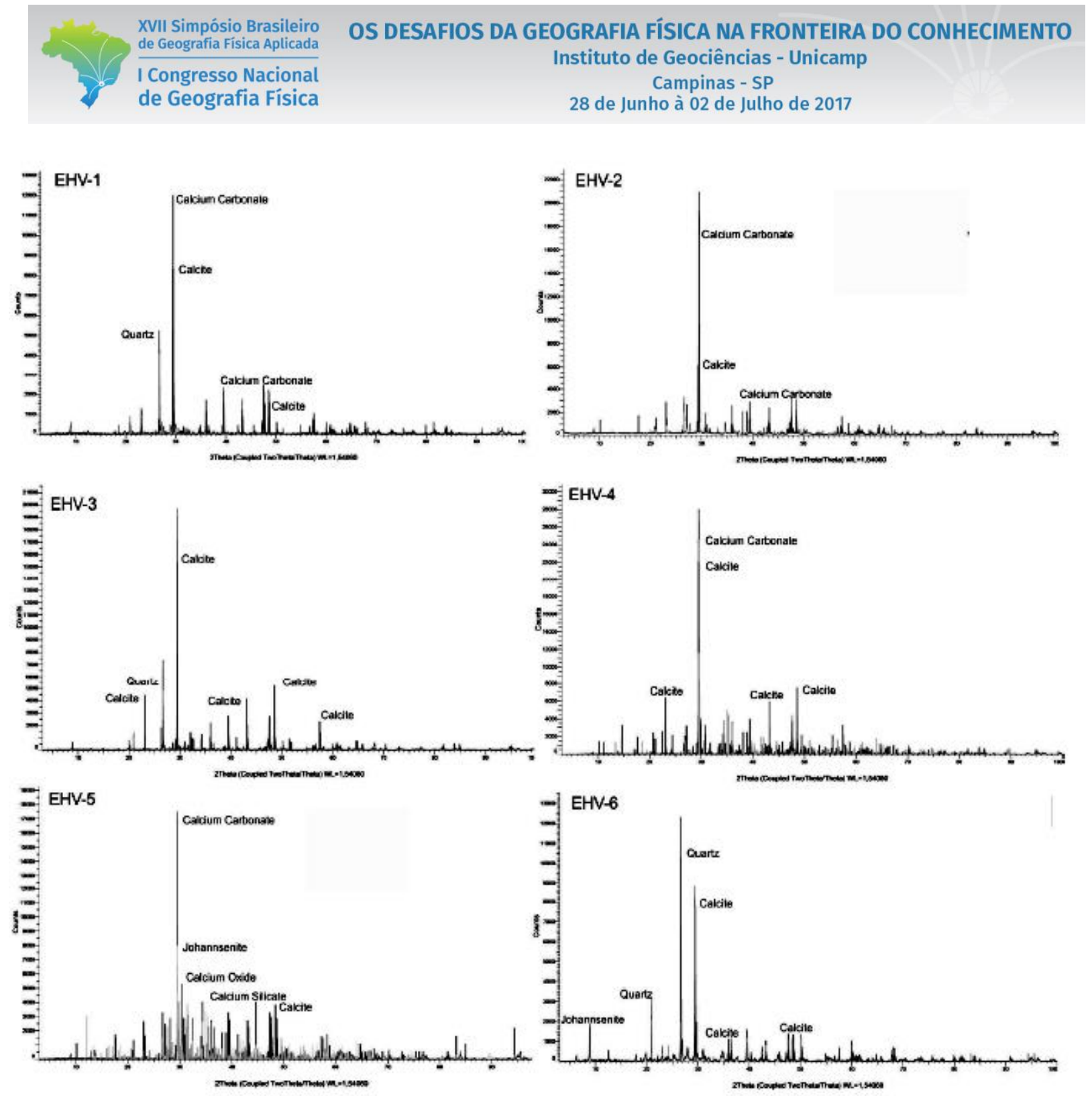

Figura 4 - Difratogramas dos materiais de fração fina.

As rochas carbonáticas são mais importantes na Cadeia Heritage do que na Cadeia Sentinel (BUGGISH e WEBERS, 1992), devido a presença da Formação Minaret. Os componentes carbonáticos e as feições sedimentares no Vale Elephant Head endossam as outras áreas carbonáticas na área. A deposição da Formação Minaret ocorreu durante o Cambriano Médio ao Tardio (WEBERS et al., 1992b).

A região estudada contém evidências geomorfológicas e sedimentares associadas ao comportamento do manto de gelo e suas antigas retrações e expansões desde o Quaternário. Investigações geomorfológicas durante o Ellsworth Mountains Project (1979-1980) concluíram que os processos de deglaciação deixaram expostas feições erosivas e sedimentares. Marcas erosivas (trimline) mapeadas por Denton et al. (1992) 
nas montanhas Ellsworth, também sugeriram que nos últimos 15 mil anos o manto de gelo da Antártica Ocidental esteve 450 a 650 metros acima da superfície atual (DENTON et al., 1992).

Estudos baseados biologia marinha sugerem que a maior parte do manto de gelo desapareceu durante os períodos interglaciais do Pleistoceno, originando uma abertura marinha entre esses dois oceanos (SCHERER et al., 1998; BARNES e HILLENBRAND, 2010; STRUGNELL et al., 2012), e que somente as áreas mais elevadas estiveram ocupadas por geleiras nos períodos interglaciais mais quentes. No entanto, estudo de Fogwill et al. (2012) revela que as morainas de gelo azul na região de Patriot Hills (70 km ao sul, aproximadamente, da geleira Union, estão presentes há pelo menos 400.000 anos, mantendo-se assim mesmo durante os períodos interglaciais mais quentes. Estudo mais recente de Hein et al. (2016), na mesma área, revela idades dos depósitos de morainas de gelo-azul mais antigas que 1.4 Ma. Isso quer dizer que em boa parte do Pleistoceno, as áreas de gelo-azul estiveram estáveis.

Considerando a localização do vale Elephant Head, as altitudes dos pontos de coletas dos sedimentos, entre 700 e 900 metros acima do nível do mar, e a espessura do manto de gelo na área da geleira Union, estimado em 1540 m (RIVERA et al., 2014), é sugerido que o material marinho depositado no vale não corresponde aos períodos interglaciais do Pleistoceno. Estudos sobre a datação dos sedimentos são necessários para refinar a história geológica e glacial do Vale Elephant Head.

\section{Considerações Finais}

Os componentes carbonáticos, a distribuição granulométrica e as feições morfométricas dos sedimentos coletados nos depósitos morânicos no Vale Elephant Head inferem o sítio como a principal área-fonte. Os depósitos e sedimentos no vale sugerem uma sucessão de processos em ambientes marinhos e nãomarinhos: sedimentação marinha provavelmente preenchendo um antigo mar raso, associado com soerguimento local devido ao efeito rebote da crosta e posterior cobertura, erosão e deposição glacial. Sedimentação carbonática e clástica (arenito e quartzito) domina o vale, expondo a natureza e composição das principais fontes de sedimentos. Conglomerados também revelam a área-fonte proximal. Condições glaciais posteriores não modificaram os sedimentos substancialmente devido às condições áridas da região e à pequena distância de transporte dos sedimentos. As principais lacunas agora se centram na escala temporal dos eventos e mais estudos são necessários para embasar as interpretações. 


\section{Agradecimentos}

A expedição Deserto de Cristal (2011/2012) foi financiada pelo CNPq e pelo Programa Antártico Brasileiro (PROANTAR). Antartic Logistics \& Expeditions deu apoio desde o transporte aéreo até o apoio logístico em terra. A Fundação de Amparo à Pesquisa do Estado do Rio de Janeiro (FAPERJ) deu suporte à pesquisa através de equipamentos de laboratório e bolsas para alunos de iniciação científica. As imagens ASTER foram oferecidas por Jorge Arigony Neto (UFRGS) e processadas no Centro Polar e Climático (UFRGS).

\section{Bibliografia}

BASU, A. Reading provenance from detrital quartz. In: Zuffa, G.G. (Ed), Provenance of Arenites. Reideo Publishing, Dordrecht, p. 231-247, 1985.

BENN D.I., BALLANTYNE C.K. Reconstructing the transport history of glaciogenic sediments - a new approach based on the covariance of clast form indices. Sedimentary Geology, 91 (1-4), p. 215-227, 1994.

BENNETT, M.R., HAMBREY, M.J., HUDDART, D. Modification of clast shape in high Arctic environments. Journal of Sedimentary Research, 67 (3), p. 550-59, 1997.

BUGGISH W., WEBERS, G.F. Facies of Cambrian carbonate rocks, Ellsworth Mountains, West Antarctica. Geological Society of America. Memoir 170, p. 81-100, 1992.

CURTIS, M.L., LOMAS, S.A. Late Cambrian stratigraphy of the Heritage Range, Ellsworth Mountains: implications for basin evolution. Antarctic Science, 11(1), p. 63-77, 1999.

CURTIS, M.L., LEAT, P.T., RILEY, T.R., STOREYA, B.C., MILLAR, I.L., RANDALL, D.E. Middle Cambrian rift-related volcanism in the Ellsworth Mountains, Antarctica: tectonic implications for the palaeo-Pacific margin of Gondwana. Tectonophysics, 304, p. 275-299, 1999.

DENTON, G.H., BOCKHEIM, J.G., RUTFORD, R.H., ANDERSEN, B.G. Glacial history of the Ellsworth Mountains, West Antarctic, in Webers, G.F., Craddock, C. e Splettstoesser, J.F. (Eds), Geology and Paleontology of the Ellsworth Mountains, West Antarctic: Boulder, Colorado, Geological Society of America, Memoir 170, 403432,1992 .

DUEBENDORFER, E.M., REES, M.N. Evidence for Cambrian deformation in the Ellsworth-Whitmore Mountains terrene, Antarctica: stratigraphic and tectonic implications. Geology, 26, p. 55-58, 1998.

EVANS, D.J.A., BENN, D.I. Facies description and the logging of sedimentary exposures. In: Evans, D.J.A. and Benn, D.I. (Eds). A Practical Guide to the Study of Glacial Sediments. Arnold, p. 11-51, 2004.

FITZGERALD, P.G., STUMP, E. Early Cretaceous Uplift of the Southern Sentinel Range, Ellsworth Mountains, West Antarctica. Recent Progress in Antarctic Earth Science: edited by Y.Yoshida et al., p. 331-340, 1992.

FOLK, R.L., WARD, W.C. Petrol Brazos River bar: a study in the significance of grain size parameters. Journal of Sedimentology, 3 (27), p. 3-26, 1957.

LUKAS, S., BENN, D.I., BOSTON, C.M., BROOK, M., CORAY, S., EVANS, D.J.A, GRAF, A., KELLERERPIRKLBAUER, A., KIRKBRID, M.P., KRABBENDAM, M., LOVELL, H., MACHIEDO, M., MILLS, S.C., NYE, K., REINARDY, B.T.I., ROSS, F.H., SIGNER, M. Clast shape analysis and clast transport paths in glacial environments: A critical review of methods and the role of lithology. Earth-Science Reviews, 121, p. 96-116, 2013.

POWERS, M.C. A new roundness scale for sedimentary particles. Journal of Sedimentary Research, 23 (2), p.117-119, 1953. 


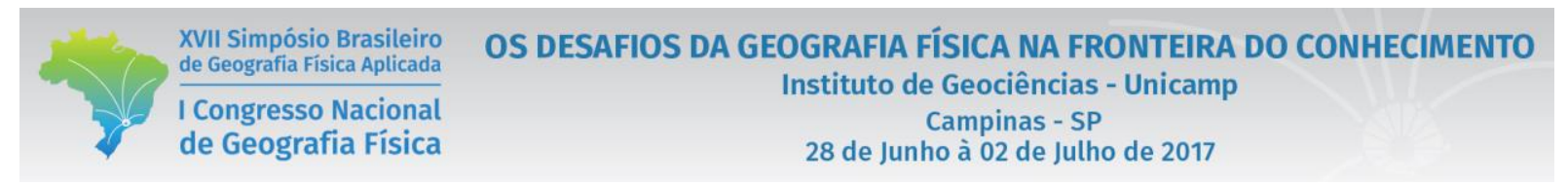

RIVERA, A., ZAMORA, R., RADA, C., WALTON, J., PROCTOR, S. Ice dynamics of Union Glacier in the Ellsworth Mountains, West Antarctica. Annals of Glaciology, 51 (55), p. 1-6, 2010.

RIVERA, A., ZAMORA, R., URIBE, J.A., JÁÑA, R., OBERREUTER, J. Recent ice dynamic and surface mass balance of Union Glacier in the West Antarctic Ice Sheet. The Cryosphere, 8, p.1445-1456, 2014.

SOUZA, C.D. Gênese, propriedades e mineralogia de solos de deserto polar da porção sul das Montanhas Ellsworth, Antártica Continental. Viçosa: UFV. Cap. 1 (Tese - Doutorado em Solos e Nutrição de Plantas), p. 338.

SPORLI, K.B. Stratigraphy of the Crashsite Group, Ellsworth Mountains, West Antarctic. In: Webers, G.F., Craddock, C., Splettstoesser, J.F. (Eds). Geology and Paleontology of the Ellsworth Mountains, West Antarctic. Geological Society of America, Memoir 170, Boulder, Colorado, p. 21-35, 1992.

STEIG, E.J., SCHNEIDER, D.P., RUTHERFORD, S.D., MANN, M.E., COMISO, J.C., SHINDELL, D.T. Warming of the Antarctic ice-sheet surface since the 1957 International Geophysical Year. Nature, 457 (7228), p. 459-462, 2009.

STOREY, B.C. Antarctic. In Selley, R.C., Cocks, R.M., Plimer, I.R. (Eds). Encyclopedia of Geology. Elsevier. 731 p., 2005.

STUMP, E. Ross Orogeny of the Transantarctic Mountains. Cambridge University Press, New York, 284 p. 1995.

VIALOV, O.S. Problematica of the Beacon Sandstone at Beacon Height West Antarctica. New Zealand Journal of Geology and Geophysics, 5, p. 718-732, 1962.

WEBERS, G.F., CRADDOCK, C., SPLETTSTOESSER, J.F. Geological history of the Ellsworth Mountains, West Antarctica, in: Webers, G.F., Craddock, C., Splettstoesser, J.F. (Eds). Geology and Palaeontology of the Ellsworth Mountains, West Antarctica. Geological Society of America, Memoir 170: p. 1-8, 1992 a.

WEBERS, G.F., POJETA, J., YOCHELSONE, L. Cambrian Mollusca from the Minaret Formation, Ellsworth Mountains, West Antarctica. In Webers, G.F., Craddock, C., Splettstoesser, J.F. (Eds) Geology und paleontology of the Ellsworth Mountains, West Antarctica. Geological Society of America, Memoir 170, p. 181-248, 1992b.

WEBERS, G.F., SPLETTSTOESSER, J.F. Review of the geology and paleontology of the Ellsworth Mountains, Antarctica. In Antarctica: A Keystone in a Changing World. Online Proceedings of the 10th ISAES, edited by A.K. Cooper and C.R. Raymond et al., USGS Open- File Report 2007-1047, Short Research Paper 107, 5 p. 2007.

WELTJE, G.J., EYNATTEN, H. Quantitative provenance analysis of sediments: review and outlook. Sedimentary Geology, 171, p. 1-11, 2004. 